\title{
Occurrence and Severity of Venous Air Embolism During Neurosurgical Procedures in Semi-Sitting Versus Supine Position
}

Franziska Magdalena Konrad ( $\sim$ franziska.konrad@uni-tuebingen.de )

University of Tübingen: Eberhard Karls Universitat Tubingen https://orcid.org/0000-0002-9259-7232

\section{Angela S Mayer}

University of Tübingen: Eberhard Karls Universitat Tubingen

\section{Lina Maria Serna-Higuita}

University of Tübingen: Eberhard Karls Universitat Tubingen

\section{Helene Hurth}

University of Tübingen: Eberhard Karls Universitat Tubingen

\section{Marcos Tatagiba}

University of Tübingen: Eberhard Karls Universitat Tubingen

Jörg Reutershan

University of Tübingen: Eberhard Karls Universitat Tubingen

\section{Peter Rosenberger}

University of Tübingen: Eberhard Karls Universitat Tubingen

\section{Berthold Drexler}

University of Tübingen: Eberhard Karls Universitat Tubingen

\section{Research Article}

Keywords: semi-sitting position, supine position, neurosurgery, vestibular schwannoma, posterior cranial fossa, transesophageal echocardiography

Posted Date: June 2nd, 2021

DOI: https://doi.org/10.21203/rs.3.rs-538659/v1

License: (c) (i) This work is licensed under a Creative Commons Attribution 4.0 International License. Read Full License

Version of Record: A version of this preprint was published at World Neurosurgery on April 1st, 2022. See the published version at https://doi.org/10.1016/j.wneu.2022.03.125. 


\section{Abstract}

Background: Patients undergoing neurosurgical procedures in the posterior cranial fossa can be placed in different positions: the semi-sitting position or the supine position. The major risk of the semi-sitting positioning is venous air embolism (VAE). However, VAEs may also occur in the supine position.

Objective: In a prospective study, we investigated the incidence of VAE based on the positioning of the patients (trial registration 553/2013B01).

Methods: In a single-center study with 137 patients, we prospectively evaluated the occurrence of VAEs in patients in the supine and semi-sitting position over the period from January 2014 to April 2015. All patients were monitored for VAE by the use of a transesophageal echocardiography (TEE).

Results: $50 \%$ of all participating patients experienced a VAE (with $56 \%$ of these patients undergoing surgery in the semi-sitting position and $11 \%$ in the prone position). $86 \%$ of the VAEs were just detected by the use of a TEE. We only observed VAEs with a decrease in $\mathrm{EtCO}_{2}$ in the semi-sitting position. However, none of the patients had any hemodynamic changes due to the VAE. We found that surgeries in patients with a preexisting intracardial shunt such as a patent foramen ovale (PFO) less likely resulted in VAEs $(42 \%$ vs. $58 \%)$.

Conclusion: The semi-sitting position with TEE monitoring and a standardized protocol, including a deep central venous line is a safe and advantageous technique, taking also account of a significant rate of VAEs. VAEs also occur in the supine position, however, less frequently.

\section{Introduction}

Pathologic processes in the posterior cranial fossa frequently induce early neurological symptoms, because this fossa encloses the cerebellum and parts of the brain stem. Over $90 \%$ of the pathologic processes of cerebellopontine angle tumors are caused by vestibular schwannomas, followed by meningiomas, epidermoid cysts and facial nerve schwannomas (4). When operating lesions of the cerebello-pontine angle, besides a complete tumor resection, anatomic and functional preservation of the cranial nerves is essential (33).

Since postoperative neurological deficits will have a major impact on the quality of life of these patients, optimizing surgical procedures seems beneficial. The cerebellopontine angle can be reached by the subtemporal, translabyrinthal or retrosigmoid approach. The latter has been shown to be advantageous due to the low level of manipulation of the brain and the possibility of reliable identification of the cranial nerves $(5,23,25)$. There are different surgical positions that enable the surgeon to access this region. Basically, patients can be treated in the supine, park-bench or in the semi-sitting position. In the semisitting position, the intracranial pressure is reduced, blood drain improved, and the view of the surgical site allows a better orientation (7). From an anesthesiological point of view, the semi-sitting position is associated with lower airway pressure, less diaphragm impairment, and good access to the endotracheal 
tube with a reduced risk of displacement compared to the prone position. Furthermore, the rate of preservation of cranial nerves is higher in the semi-sitting position compared to other surgical positions $(3,27)$. The semi-sitting position mainly differs from the sitting position with regard to the level of the legs (30). In the semi-sitting position, the torso and the legs are elevated, whereas the hips are inflected at a maximum of $90^{\circ}$. Accordingly, the fundament is the deepest point with the feet at least at ear level. In this position, the anesthesiologists can compress the jugular veins in case of a VAE, which is - in addition to arterial hypotension - the most serious complication of this position $(7,10)$.

Many neurosurgeons prefer the semi-sitting position to remove particular large lesions of the posterior fossa, whereas anesthesiologists may be concerned about patients developing a VAE. There are variations across countries; for example in the UK, it is the neurosurgeon who usually decides on the patient's position (11), whereas in Japan, anesthesiologists usually decide against the (semi-)sitting position (12). Over many years, there had been a decrease in the use of the semi-sitting position in neurosurgery in fear of VAEs $(24,32)$. In recent years increasing interest resurged. Therefore, we investigated the impact of the position of the patient - supine versus semi-sitting - on VAEs during neurosurgical interventions in a prospective study. In particular, we investigated the clinical impact and the severity of the occurrence of VAEs in both positions in patients with and without a pre-existing intracardial shunt such as a PFO.

\section{Methods And Material}

\section{Study design}

This prospective single-center study was conducted in the Department of Anesthesiology and Intensive Care Medicine and the Department of Neurosurgery at the University Hospital Tübingen. Patients were included over the period from January 2014 to April 2015 in accordance with the Ethics Committee of the University of Tübingen (553/2013B01).

After having received their informed consent, we included adult patients requiring an elective operation in the posterior fossa of the cranium. Patients with existing contra-indications against transesophageal echocardiography (TEE) were excluded. We also excluded patients with revision surgeries and pregnant women.

\section{Preoperative patient preparation}

All patients were informed about this study as part of our routine patient information on general anesthesia. To detect a PFO or other right-left shunts, both a transthoracic echocardiography with simultaneous valsalva maneuver and an injection of a contrast medium (gelafundin ${ }^{\circledR}$ ) were performed in 
every patient. We ascertained and documented the neurological status with the focus on detecting increased intracranial pressure and pre-existing cranial nerve deficits such as facial paralysis or loss of hearing. At the end, we performed a general physical examination, including biometric data, blood pressure, body weight etc.

\section{Intraoperative procedure}

All patients received standard monitoring, consisting of ECG, oxygen saturation, and noninvasive blood pressure. Induction of anesthesia was performed using $0.3 \mu \mathrm{g} / \mathrm{kgBW}$ sufentanil up to a maximum of 50 $\mu \mathrm{g}, 1.5-2.5 \mathrm{mg} / \mathrm{kgBW}$ propofol and $0.6 \mathrm{mg} / \mathrm{kgBW}$ rocuronium. After endotracheal intubation, anesthesia was maintained by $5 \mathrm{mg} / \mathrm{kg} / \mathrm{h}$ propofol and $0.5 \mu \mathrm{g} / \mathrm{kg} / \mathrm{min}$ remifentanil without a bolus administration so as not to affect the neuromonitoring. Patients were ventilated at a tidal volume of $6-8 \mathrm{ml} / \mathrm{kgBW}$ and at a positive end-expiratory pressure (PEEP) at a maximum of $5 \mathrm{cmH}_{2} \mathrm{O}$ to achieve normoventilation with an end-tidal $\mathrm{CO}_{2}$ of $34-36 \mathrm{mmHg}$. All patients received an antibiotic prophylaxis with cefuroxime. If advised by the neurosurgeon, some of the patients received $20 \mathrm{mg}$ dexamethasone to prevent postoperative edema.

Preoperative preparation included: two peripheral venous accesses, invasive blood pressure monitoring, temperature measurement, a heating cover, and a urinary catheter. We placed the pressure transducer at ear level to measure the cerebral arterial pressure at the level of the surgical site and kept the mean arterial pressure at $80 \mathrm{mmHg}$ using noradrenalin. Then, we placed a central venous catheter with a tip of approximately 1 to $2 \mathrm{~cm}$ in the right atrium and performed a transesophageal echocardiography (TEE) monitoring throughout the operation to detect a VAE. Therefore, we placed the probe in an angle so as to have a bicaval view on the right atrium or to display the right ventricular inflow and outflow. Besides the common side stream capnography, we added a main stream capnography to even detect the slightest changes in $\mathrm{EtCO}_{2}$. We used neuromonitoring to derive sensory, motor and auditory evoked potentials to monitor the integrity of the neural innervation of the mimetic muscles and the auditory function.

After we had induced general anesthesia, we positioned the patients for the operation, using a Mayfield ${ }^{\circledR}$ skull clamp to fixate the head. Then, an experienced team of surgeons, neuro-anesthesiologists and nurses placed the patients in either the supine or the semi-sitting position in accordance with strict standards. The decision on which position to choose was made by the neurosurgeon depending on the tumor size and entity and was approved by the anesthesiologist preoperatively.

\section{Acquisition of data}

We recorded all vital and ventilation parameters as well as any administration of medication using the patient management software CareView and ICCA (both from Philips ${ }^{\circledR}$ Healthcare), respectively. The anesthesiologists documented any air entrance (i.e. VAE) on a special documentation form, including the following parameters: preexisting PFO or any other intracardial shunt, visible air bubbles in the TEE monitoring, time of air detection, performed aspiration of air via the central venous line, $\mathrm{EtCO}_{2}$ (end-tidal), 
$\Delta \mathrm{CO}_{2}$ (delta), mean arterial pressure and central venous pressure at the time of the VAE. Furthermore, we documented the possible cause of the air embolism if the surgical source of the VAE could be detected after compression of the jugular veins.

We collected all biometric data (age, gender, BMI, ASA-classification), the indication and duration of the surgical procedure, the localization of the tumor and the intraoperative positioning. We classified both the localization and the extension of the vestibular schwannoma according to Matthies et al. (20).

\section{VAE grading and management}

We graded the VAE according to the "Tübingen VAE grading scale" as previously described (7). The Tübingen VAE score classifies stages of air embolism in six grades from 0 (no air bubbles in TEE) to 5 (VAE demanding cardiopulmonary resuscitation). This classification is summarized in Table 1. In case of multiple air embolisms during an operation, only the most severe grade was evaluated. The anesthesiologists immediately informed the surgeons of any air bubbles in the TEE, which was followed by compressing both jugular veins. Then, the surgeons occluded any open veins in their field.

\section{Statistical analysis}

We performed a statistical analysis, using SPSS Statistics ${ }^{\circledR}$ Version 22, IBM, with $\mathrm{P}<0.05$ considered as statistically significant. Descriptive analysis was performed, categorical variables were described using absolute and relative frequency. Numerical variables were set out as means and standard deviation or medians, and interquartile ranges (IQR) according to the distribution of their data. Normality of the distribution was assessed by investigating kurtosis, skewness as well as QQ graphs, Box plots and Histograms. Bivariate analysis to check differences between groups was performed. $\mathrm{Chi}^{2}$ test of Fisher's exact test for categorical variables was used as appropriate. Independent-samples t-test were used to compare quantitative variables that were approximately normally distributed, while Mann-Whitney test was performed for skewed variables. Cochraine test was used to evaluate dichotomous dependent variables.

\section{Results}

\section{Patients}

We included 137 patients in the present study, of which $14 \%$ (19 patients) were operated in the supine position and $86 \%$ (118) in the semi-sitting position. The statistically analyzed biometric properties such as the average age and body mass index, did not show any difference in these two groups (see Table 2). Neither did the incidence of any preexisting intracardial shunts differ in these groups.

\section{Surgery}

We did not find any significant difference in the various indications for the operation in these groups except for tumor size (Table 3). The most frequent indication for surgery in both groups was a vestibular 
schwannoma (77\%). However, the size of the vestibular schwannoma had a significant impact on which position we chose for the patient (Table 3 ). Small schwannomas $(T 1, T 2)$ were mainly removed in the supine position, larger schwannomas $(\mathrm{T} 3, \mathrm{~T} 4)$ were almost always removed in the semi-sitting position. The duration of the surgical procedure itself took significantly longer in the semi-sitting position compared to those procedures taking place in the supine position (Table 3 ), reflecting the different tumor sizes.

\section{Perioperative venous air embolism}

VAEs occurred in both positions (Table 3), but VAEs occurred significantly more frequently in patients placed in the semi-sitting position ( $56 \%$ vs. $11 \%$ ). We documented the vital parameters such as MAP and CVP at each episode of VAE. At the onset of the first VAE, we found that MAP was significantly lower in the semi-sitting position compared to the prone group (Table 3). The central venous pressure did not differ between these groups. We detected approximately $26 \%$ of the VAEs during the first 30 minutes of the operation, therefore, we found that this phase of the procedure (craniotomy) entailed the highest risk in both positioning groups (Table 4).

Although VAE occurred more frequently in semi-sitting than in supine position, the severity of the VAE did not differ between these positioning groups (Table 3 ). The vast majority (86\%) of the VAEs was grade I and therefore did not lead to any changes in the vital parameters. We observed a grade II VAE in one supine-positioned patient (5.3\%) as well as in five patients in the semi-sitting position. Nevertheless, grade III (air bubbles and a decrease in $\mathrm{EtCO}_{2}>3 \mathrm{mmHg}$ ), occurred in four patients in the semi-sitting position. We carried out an aspiration of air only in one patient in the semi-sitting position.

We operated on $20 \%$ of the patients with a preexisting venous-arterial shunt in the supine position, with $80 \%$ undergoing the procedure in the semi-sitting position. We found that irrespective of the positioning, patients with a preexisting venous-arterial shunt developed fewer VAEs compared to patients without a shunt (Table 5).

\section{Discussion}

In the present study, we evaluated the impact of the positioning of patients on the occurrence of a VAE during neurosurgical operations in the posterior cranial fossa. Over a period of 15 months, we included 137 patients in our study, of which 118 patients underwent surgery in the semi-sitting position and 19 in the supine position. Lindroos et al. have gained extensive experience by having used the semi-sitting position for almost 20 years $(14,17,26,31)$. The semi-sitting position optimizes the operation situation for the surgeon in that it causes a drainage of liquor and blood from the surgical field by gravity, decreases intracranial pressure and enables the monitoring of the motoric response of patients to cranial nerve stimulation $(14,17,26,32)$. In the semi-sitting position, the venous pressure at heart level may be below the atmospheric pressure, which puts patients at a potential risk for rapid air inflow from the 
surgical field into the venous circulation, i.e., causing a VAE (13). For fear of VAEs, some institutions refuse to use this position $(7,24)$.

The most frequent indication for surgery in a semi-sitting position was a vestibular schwannoma in this study, which is in accordance with the literature (24). At our institution, we almost exclusively operated on larger schwannomas that reached the fossa posterior (T3) and compressed the brain stem (T4) in the semi-sitting position, since it provides easier access and a total resection is more likely (27). Furthermore, Link et al. showed an increase in the quality of life in patients who underwent a complete resection (15). In our series, the greater spread of the tumor in patients operated in the semi-sitting position explains the prolonged duration of the procedure in these cases.

The position for a neurosurgical procedure depends on the surgeon's experience and preferred operating technique (28). A metaanalysis of 1792 patients operated in the sitting positions found a low overall complication rate of $1.45 \%$ (9). Another institution reported that 192 patients had been operated on the posterior cranial fossa, of which 92 underwent the surgery in the sitting position and 100 in the horizontal position. The operating position had to be changed in 6 patients due to hemodynamic instability or uncontrolled VAE. However, $11 \%$ of patients operated in the horizontal position also showed severe VAE (24). The high rate of hemodynamically relevant VAE in that series compared to our data might be explained due to the lower level of the legs in the sitting position compared to the semi-sitting position used in our institution.

In the present study, we monitored all patients by the use of a TEE. In accordance with the literature, $56 \%$ of the patients in the semi-sitting position and $11 \%$ in the supine position experienced a VAE in the present study $(3,22,24)$. So far, the use of a TEE is not the gold standard for monitoring patients undergoing such procedures. Some institutions diagnose VAEs only based on a change in $\operatorname{EtCO}_{2}(2,21)$; Bithal et al. could not derive any benefits from the use of TEE monitoring either (1). If a TEE is used, VAEs can often be diagnosed before any hemodynamic changes are caused. By applying these techniques, many institutions have reported a decline of severe VAEs in the semi-sitting position $(8-10,14,22)$.

In the present study, roughly $25 \%$ of the VAEs occurred during the first 30 minutes of the procedure, e.g., a typically critical period for injuries to the venae emissariae or small venous entry points in the skull or musculature. Other authors also detected VAEs at the beginning of their operations, leading them to assume that there is a link between the craniotomy and the $\operatorname{VAE}(10,29)$.

Severe VAEs in the semi-sitting position with hemodynamic and respiratory changes were reported to occur in $3.3 \%$ (8) and $1.06 \%$ (9), but also in up to 50\% (32). The first two incident rates described in retrospective studies, were based on intraoperative documentation. However, in emergency cases, the documentation is usually to be completed afterwards. The $50 \%$ of severe VAEs were derived from a prospective study, comparing a $30^{\circ}$ and a $45^{\circ}$ positioning of the head. In the latter positioning, the head marked the highest point of the patient. In our present study, $6 \%$ of all patients had a VAE grade III, defined by a decrease in $\mathrm{EtCO}_{2}$. There was no grade IV VAE, which would include hemodynamic changes. 
The main reason for the lower rate of a clinically-relevant VAE may be the combination of the patient's leg elevation combined with the patient's head bent toward the sternum, which most likely causes a decrease in the venous return to the right heart (32).

In some institutions, patients with a PFO are excluded from the semi-sitting position (10). However, autopsy studies revealed the incidence of a PFO in the general population as high as $35 \%(16,18)$. At our institution, all patients who are preoperatively diagnosed with an intracardial shunt are discussed by a team of experts for their individually best solution. If these patients are still scheduled to have surgery in the semi-sitting position, they are informed of the potential consequences of a VAE, but these patients usually also suffer from larger tumors. However, in the present study, a pre-existing right-left shunt was associated with a lower risk of developing a VAE. This finding may be attributed to the fact that both the anesthesiologist and the neurosurgeon pay particular attention to avoiding any VAE in this specific situation. In addition, our institution has become quite experienced at this operation method over the past ten years by treating around 100 cases each year. To our knowledge, no patient at our institution has ever experienced any persisting damage by developing a VAE in the semi-sitting position. Nevertheless, once there is air in the right atrium in case of a PFO or other right-left shunts, there is no additional warning parameter, which means that any air embolism could lead to potentially deleterious neurological consequences $(10,19,22)$. Hence, in our opinion, patients with a PFO or right-left shunt should be informed about this potentially higher risk. However, we do not consider a PFO to be a definite contraindication for the semi-sitting position. Other prospective studies came to similar conclusions (6, 7).

This study demonstrates that VAEs also occur in the supine position. In addition, patients with an existing venous-arterial shunt were less likely to experience a VAE regardless of their positioning, which suggests that the particular attention neurosurgeons and anesthesiologists direct to these patients to avoid VAEs may also have an impact.

\section{Conclusions}

The semi-sitting position is a safe procedure for an experienced team of neurosurgeons and neuroanesthesiologists, even in cases of preexisting venous-arterial shunts, if they employ a standardized protocol. Finally, VAEs are more common in the semi-sitting position, but also occur in the supine position.

\section{Declarations}

\section{Funding:}

This study was funded by the German research foundation grant RE KO 4280/1-1 (to F. Konrad).

\section{Conflict of interests:}


There are no conflicts of interests for any of the authors.

\section{Availability of data and material:}

All data is available.

\section{Code availability:}

Not applicable.

\section{Ethics approval:}

Patients were included in accordance with the Ethics Committee of the University of Tübingen (553/2013B01).

\section{Consent to participate:}

All authors have read and approved this manuscript.

\section{Consent for publication}

The publication is not under consideration elsewhere.

\section{Authors' contributions}

Franziska M Konrad analyzed the data, interpreted the data and wrote the manuscript. Angela S Mayer collected the data. Lina Serna Higuita analyzed the data. Helene Hurth, Marcos Tatagiba, Jörg Reutershan, Peter Rosenberger and Berthold Drexler edited the manuscript substantially, helped collecting the data and interpreting it.

\section{Acknowledgments}

This study was funded by the German research foundation grant RE KO 4280/1-1 (to F. Konrad). The authors gratefully thank Karlheinz Decker, MD for consistently supporting our work.

\section{References}

1. Bithal P, Dash HH, Vishnoi N, Chaturvedi A (2003) Venous air embolism: does the site of embolism influence the hemodynamic changes? Neurol India 51:370-372

2. Bithal PK, Pandia MP, Dash HH, Chouhan RS, Mohanty B, Padhy N (2004) Comparative incidence of venous air embolism and associated hypotension in adults and children operated for neurosurgery in the sitting position. Eur J Anaesthesiol 21:517-522

3. Black S, Ockert DB, Oliver WC Jr, Cucchiara RF (1988) Outcome following posterior fossa craniectomy in patients in the sitting or horizontal positions. Anesthesiology 69:49-56 
4. Bonneville F, Savatovsky J, Chiras J (2007) Imaging of cerebellopontine angle lesions: an update. Part 1: enhancing extra-axial lesions. Eur Radiol 17:2472-2482

5. Ebner FH, Tatagiba M (2019) [Update on diagnostics and microsurgical treatment of vestibular schwannoma]. Nervenarzt 90:578-586

6. Engelhardt M, Folkers W, Brenke C, Scholz M, Harders A, Fidorra H, Schmieder K (2006) Neurosurgical operations with the patient in sitting position: analysis of risk factors using transcranial Doppler sonography. Br J Anaesth 96:467-472

7. Feigl GC, Decker K, Wurms M, Krischek B, Ritz R, Unertl K, Tatagiba M (2014) Neurosurgical procedures in the semisitting position: evaluation of the risk of paradoxical venous air embolism in patients with a patent foramen ovale. World Neurosurg 81:159-164

8. Ganslandt O, Merkel A, Schmitt H, Tzabazis A, Buchfelder M, Eyupoglu I, Muenster T (2013) The sitting position in neurosurgery: indications, complications and results. a single institution experience of 600 cases. Acta Neurochir (Wien) 155:1887-1893

9. Himes BT, Mallory GW, Abcejo AS, Pasternak J, Atkinson JLD, Meyer FB, Marsh WR, Link MJ, Clarke MJ, Perkins W, Van Gompel JJ (2017) Contemporary analysis of the intraoperative and perioperative complications of neurosurgical procedures performed in the sitting position. $\mathrm{J}$ Neurosurg 127:182188

10. Jadik S, Wissing H, Friedrich K, Beck J, Seifert V, Raabe A (2009) A standardized protocol for the prevention of clinically relevant venous air embolism during neurosurgical interventions in the semisitting position. Neurosurgery 64:533-538; discussion 538-539

11. Jurgens S, Basu S (2014) The sitting position in anaesthesia: old and new. Eur J Anaesthesiol 31:285-287

12. Kida H, Nishikawa N, Matsunami K, Kawahito M, Ota M, Miyao S (2000) [Sitting position in the neurosurgery: the results of a questionnaire sent to neurosurgeons of medical colleges]. Masui 49:566-569

13. King MB, Harmon KR (1994) Unusual forms of pulmonary embolism. Clin Chest Med 15:561-580

14. Lindroos AC, Niiya T, Randell T, Romani R, Hernesniemi J, Niemi T (2010) Sitting position for removal of pineal region lesions: the Helsinki experience. World Neurosurg 74:505-513

15. Link MJ, Lund-Johansen M, Lohse CM, Driscoll CLW, Myrseth E, Tveiten OV, Carlson ML (2018) Quality of Life in Patients with Vestibular Schwannomas Following Gross Total or Less than Gross Total Microsurgical Resection: Should We be Taking the Entire Tumor Out? Neurosurgery 82:541547

16. Lobato EB, Black S, De Soto H (1997) Venous air embolism and selective denervation for torticollis. Anesth Analg 84:551-553

17. Malis LI (2001) Nuances in acoustic neuroma surgery. Neurosurgery 49:337-341

18. Mammoto T, Hayashi Y, Ohnishi Y, Kuro M (1998) Incidence of venous and paradoxical air embolism in neurosurgical patients in the sitting position: detection by transesophageal echocardiography. Acta Anaesthesiol Scand 42:643-647 
19. Matjasko J, Petrozza P, Cohen M, Steinberg P (1985) Anesthesia and surgery in the seated position: analysis of 554 cases. Neurosurgery 17:695-702

20. Matthies C, Samii M (1997) Management of 1000 vestibular schwannomas (acoustic neuromas): clinical presentation. Neurosurgery 40:1-9; discussion 9-10

21. Muley SS, Saini SS, Dash HH, Bithal PK (1990) End tidal carbon dioxide monitoring for detection of venous air embolism. Indian J Med Res 92:362-366

22. Papadopoulos G, Kuhly P, Brock M, Rudolph KH, Link J, Eyrich K (1994) Venous and paradoxical air embolism in the sitting position. A prospective study with transoesophageal echocardiography. Acta Neurochir (Wien) 126:140-143

23. Rajput MSA, Ahmad AN, Arain AA, Adeel M, Akram S, Awan MS, Bari ME (2018) Preservation of Hearing and Facial Nerve Function with the Microsurgical Excision of Large Vestibular Schwannomas: Experience with the Retrosigmoid Approach. Cureus 10:e3684

24. Rushatamukayanunt P, Seanho P, Muangman S, Raksakietisak M (2016) Severe Venous Air Embolism Related to Positioning in Posterior Cranial Fossa Surgery in Siriraj Hospital. J Med Assoc Thai 99:511-516

25. Samii M, Gerganov V, Samii A (2006) Improved preservation of hearing and facial nerve function in vestibular schwannoma surgery via the retrosigmoid approach in a series of 200 patients. $J$ Neurosurg 105:527-535

26. Samii M, Matthies C (1997) Management of 1000 vestibular schwannomas (acoustic neuromas): the facial nerve-preservation and restitution of function. Neurosurgery 40:684-694; discussion 694 $-685$

27. Scheller C, Rampp S, Tatagiba M, Gharabaghi A, Ramina KF, Ganslandt O, Bischoff B, Matthies C, Westermaier T, Pedro MT, Rohde V, von Eckardstein K, Strauss C. A critical comparison between the semisitting and the supine positioning in vestibular schwannoma surgery: subgroup analysis of a randomized, multicenter trial. J Neurosurg 2019: 1-8

28. Silk PS, Lane JI, Driscoll CL (2009) Surgical approaches to vestibular schwannomas: what the radiologist needs to know. Radiographics 29:1955-1970

29. Tateishi H (1972) Prospective study of air embolism. Br J Anaesth 44:1306-1310

30. Tausk HC, Miller R (1983) Anesthesia for posterior fossa surgery in the sitting position. Bull N Y Acad Med 59:771-783

31. Tempelhoff R, Williams EL, Vollmer DG (1992) Is the "kneeling" prone position as dangerous as the sitting position for the development of venous air embolism? Anesth Analg 75:467-468

32. Ture H, Harput MV, Bekiroglu N, Keskin O, Koner O, Ture U (2018) Effect of the degree of head elevation on the incidence and severity of venous air embolism in cranial neurosurgical procedures with patients in the semisitting position. J Neurosurg 128:1560-1569

33. Zanoletti E, Mazzoni A, Martini A, Abbritti RV, Albertini R, Alexandre E, Baro V, Bartolini S, Bernardeschi D, Bivona R, Bonali M, Borghesi I, Borsetto D, Bovo R, Breun M, Calbucci F, Carlson ML, Caruso A, Caye-Thomasen P, Cazzador D, Champagne PO, Colangeli R, Conte G, D'Avella D, Danesi G, Deantonio 
L, Denaro L, Di Berardino F, Draghi R, Ebner FH, Favaretto N, Ferri G, Fioravanti A, Froelich S, Giannuzzi A, Girasoli L, Grossardt BR, Guidi M, Hagen R, Hanakita S, Hardy DG, Iglesias VC, Jefferies S, Jia H, Kalamarides M, Kanaan IN, Krengli M, Landi A, Lauda L, Lepera D, Lieber S, Lloyd SLK, Lovato A, Maccarrone F, Macfarlane R, Magnan J, Magnoni L, Marchioni D, Marinelli JP, Marioni G, Mastronardi V, Matthies C, Moffat DA, Munari S, Nardone M, Pareschi R, Pavone C, Piccirillo E, Piras G, Presutti L, Restivo G, Reznitsky M, Roca E, Russo A, Sanna M, Sartori L, Scheich M, Shehata-Dieler W, Soloperto D, Sorrentino F, Sterkers O, Taibah A, Tatagiba M, Tealdo G, Vlad D, Wu H, Zanetti D (2019) Surgery of the lateral skull base: a 50-year endeavour. Acta Otorhinolaryngol Ital 39:S1-S146

\section{Tables}

Table 1

Tübingen venous air embolism grading scale

\section{Scale}

Grade no air embolism

0

Grade air bubbles visible in the TEE

I

Grade air bubbles visible with a decrease in $\mathrm{EtCO}_{2} \leq 3 \mathrm{mmHg}$

II

Grade air bubbles visible with a decrease in $\mathrm{EtCO}_{2}>3 \mathrm{mmHg}$

III

Grade air bubbles visible with a decrease in $\mathrm{EtCO}_{2}>3 \mathrm{mmHg}$ and decrease of MAP $\geq 20 \%$ or

IV increase in heart rate $\geq 40 \%$ (or both)

Grade grade 4 causing hemodynamic instability requiring cardiopulmonary resuscitation V 
Table 2

Demographics and characteristics of the study population $(n=137)$

\begin{tabular}{|c|c|c|c|}
\hline & Supine position & Semi-sitting position & p-value \\
\hline Patients n (\%) & $19(14 \%)$ & $118(86 \%)$ & \\
\hline Age in years mean $\pm S D($ min-max $)$ & $51 \pm 12(26-72)$ & $49 \pm 14(21-80)$ & $0.546^{*}$ \\
\hline $\begin{array}{l}\text { Gender } \\
\text { Male n (\%) } \\
\text { Female n (\%) }\end{array}$ & $\begin{array}{l}7(36.8 \%) \\
12(63.2 \%)\end{array}$ & $\begin{array}{l}54(45.8 \%) \\
64(54.2 \%)\end{array}$ & $0,62^{\#}$ \\
\hline $\mathrm{BMI}\left[\mathrm{kg} / \mathrm{m}^{2}\right]$ mean $\pm \mathrm{SD}(\min -\mathrm{max})$ & $25 \pm 5(18-37)$ & $25 \pm 4(16-42)$ & $0.74 *$ \\
\hline $\begin{array}{l}\text { ASA status } \\
\text { In }(\%) \\
\text { II } n(\%) \\
\text { III n (\%) } \\
\text { IV n (\%) }\end{array}$ & $\begin{array}{l}3(15.8 \%) \\
15(78.9 \%) \\
1(5.3 \%) \\
0(0 \%)\end{array}$ & $\begin{array}{l}19(16.1 \%) \\
92(78 \%) \\
6(5.1 \%) \\
1(0.8 \%)\end{array}$ & $0.99^{\circ}$ \\
\hline Preexisting cardial venous-arterial shunt n (\%) & $6(31.6 \%)$ & $24(20.3 \%)$ & $0.207^{\#}$ \\
\hline
\end{tabular}


Table 3

Operation details $(n=137)$

\begin{tabular}{|c|c|c|c|}
\hline & $\begin{array}{l}\text { Supine } \\
\text { position }\end{array}$ & $\begin{array}{l}\text { Semi-sitting } \\
\text { position }\end{array}$ & p-value \\
\hline $\begin{array}{l}\text { OP indication } \\
\text { vestibular schwannoma n (\%) } \\
\text { cavernoma } \\
\text { ependymoma } \\
\text { epidermoid } \\
\text { meningioma } \\
\text { others }\end{array}$ & $\begin{array}{l}13 \\
0 \\
0 \\
0 \\
5 \\
1\end{array}$ & $\begin{array}{l}92 \\
3 \\
1 \\
2 \\
16 \\
4\end{array}$ & $0.562^{\#}$ \\
\hline $\begin{array}{l}\text { Size of the vestibular schwannoma (n } \\
=104) \\
\text { T1 } \mathrm{n}(\%) \\
\text { T2 } \mathrm{n}(\%) \\
\text { T3 n }(\%) \\
\text { T4 } \mathrm{n}(\%)\end{array}$ & $\begin{array}{l}3(23.1 \%) \\
8(61.5 \%) \\
2(15.4 \%) \\
0(0 \%)\end{array}$ & $\begin{array}{l}0(0 \%) \\
5(5.5 \%) \\
42(46.2 \%) \\
44(48.4 \%)\end{array}$ & $<0.001^{\#}$ \\
\hline Duration of the operation [h] & $3.3 \pm 1.2$ & $4 \pm 1.2$ & $0.032^{+}$ \\
\hline $\begin{array}{l}\text { VAE yes } n(\%) \\
\text { VAE not } n(\%)\end{array}$ & $\begin{array}{l}2(10.5 \%) \\
17(89.5 \%)\end{array}$ & $\begin{array}{l}66(55.9 \%) \\
52(44.1 \%)\end{array}$ & $<0.001^{\#}$ \\
\hline MAP at first VAE [mmHg] mean (SD) & $91 \pm 6$ & $77 \pm 9$ & $0.006^{+}$ \\
\hline $\begin{array}{l}\text { Severity of VAE* }(n=68) \\
\text { I } n(\%) \\
\text { II } n(\%) \\
\text { III } n(\%)\end{array}$ & $\begin{array}{l}1(50 \%) \\
1(50 \%) \\
0\end{array}$ & $\begin{array}{l}57(86 \%) \\
5(8 \%) \\
4(6 \%)\end{array}$ & \\
\hline
\end{tabular}

Table 4

Percentage of venous air embolism by the time period of surgery $(n=$

137)

\begin{tabular}{|lll|}
\hline & Both positions & p-value \\
\hline Time until venous air embolism [min] & & $<0.001^{\wedge}$ \\
$0-30 \mathrm{n}(\%)$ & $35(25.5 \%)$ & \\
$31-60 \mathrm{n}(\%)$ & $12(8.8 \%)$ & \\
$61-90 \mathrm{n}(\%)$ & $11(8 \%)$ & \\
$91-120 \mathrm{n}(\%)$ & $4(2.9 \%)$ & \\
$121-150 \mathrm{n}(\%)$ & $2(1.5 \%)$ & \\
$151-180 \mathrm{n}(\%)$ & $2(1.5 \%)$ & \\
$>180 \mathrm{n}(\%)$ & $2(1.5 \%)$ & \\
\hline
\end{tabular}




\section{Table 5}

Percentage of patients with VAE vs. pre-existing venous-arterial shunt $(n=137)$.

\begin{tabular}{|c|c|c|c|c|}
\hline & $\mathbf{n}$ & Supine position & Semi-sitting position & p-value \\
\hline $\begin{array}{l}\text { Pre-existing venous arterial shunt yes } \\
\text { VAE not } n(\%) \\
\text { VAE yes } n(\%)\end{array}$ & 30 & $\begin{array}{l}6(100 \%) \\
0(0 \%)\end{array}$ & $\begin{array}{l}14(58.3 \%) \\
10(41.7 \%)\end{array}$ & $0.074^{\#}$ \\
\hline $\begin{array}{l}\text { No pre-existing venous arterial shunt } \\
\text { VAE not } n(\%) \\
\text { VAE yes } n(\%)\end{array}$ & 107 & $\begin{array}{l}11(84.6 \%) \\
2(15.4 \%)\end{array}$ & $\begin{array}{l}38 \text { (40.4\%) } \\
56(59.6 \%)\end{array}$ & $0.006^{\#}$ \\
\hline
\end{tabular}

